\title{
The 51-63 base pair of tRNA confers specificity for binding by EF-Tu
}

\author{
LEE E. SANDERSON and OLKE C. UHLENBECK \\ Department of Biochemistry, Molecular Biology and Cell Biology, Northwestern University, Evanston, Illinois 60208, USA
}

\begin{abstract}
Elongation factor Tu (EF-Tu) exhibits significant specificity for the different elongator tRNA bodies in order to offset its variable affinity to the esterified amino acid. Three X-ray cocrystal structures reveal that while most of the contacts with the protein involve the phosphodiester backbone of tRNA, a single hydrogen bond is observed between the Glu390 and the amino group of a guanine in the 51-63 base pair in the T-stem of tRNA. Here we show that the Glu390Ala mutation of Thermus thermophilus EF-Tu selectively destabilizes binding of those tRNAs containing a guanine at either position 51 or 63 and that mutagenesis of the 51-63 base pair in several tRNAs modulates their binding affinities to EF-Tu. A comparison of Escherichia coli tRNA sequences suggests that this specificity mechanism is conserved across the bacterial domain. While this contact is an important specificity determinant, it is clear that others remain to be identified.
\end{abstract}

Keywords: minor groove interaction; RNA-protein interaction; base specific

\section{INTRODUCTION}

Elongation factor $\mathrm{Tu}(\mathrm{EF}-\mathrm{Tu})$ is the bacterial G-protein responsible for catalyzing the efficient delivery of aminoacyl-tRNA (aa-tRNA) to the ribosome during translation. When complexed with GTP, EF-Tu binds elongator aa-tRNA with high affinity to form a ternary complex that subsequently binds the ribosomal entry site. EF-Tu binds all elongator aa-tRNAs with roughly equivalent affinities (Louie et al. 1984; Ott et al. 1990b). However, subsequent studies using misacylated tRNAs have shown that EF-Tu exhibits substantial specificity for both the tRNA body and the esterified amino acid (LaRiviere et al. 2001; Asahara and Uhlenbeck 2002; Dale et al. 2004). The affinities for the tRNA bodies and amino acids are arranged in such a way that correctly aminoacylated tRNA binds EF-Tu uniformly, presumably to compensate for the variable thermodynamic contribution of the esterified amino acids. Thus, the binding of EF-Tu to tRNA is sequence specific. At this point, the mechanism by which EF-Tu generates specificity for its wide range of elongator tRNA substrates is unknown.

The binding affinities of three Escherichia coli aa-tRNAs and one yeast aa-tRNA to 20 point mutations of the tRNA

Abbreviations: EF-Tu, elongation factor Tu; aa-tRNA, aminoacyl-tRNA. Reprint requests to: Olke C. Uhlenbeck, Department of Biochemistry, Molecular Biology and Cell Biology, Northwestern University, 2205 Tech Drive, Hogan 2-100, Evanston, IL 60208, USA; e-mail: o-uhlenbeck@ northwestern.edu; fax: (847) 491-5444.

Article published online ahead of print. Article and publication date are at http://www.rnajournal.org/cgi/doi/10.1261/rna.485307. binding cleft of $T$. thermophilus EF-Tu revealed that the specificity of the protein for the different tRNA bodies was due to the varying thermodynamic contribution of only five amino acids (Sanderson and Uhlenbeck 2007b). A comparison of these data to the X-ray cocrystal structures of Thermus aquaticus EF-Tu bound to yeast Phe-tRNA ${ }^{\text {Phe }}$ reveals that three of the five "specificity" amino acids contact sites on the tRNA that vary in sequence among the four test aa-tRNAs. This suggested that at least part of the mechanism of specificity involved a discrete set of amino acids that differentially bind certain tRNA sequence elements. The largest specificity effect is the Glu390Ala mutation, which caused a $>2.8 \mathrm{kcal} / \mathrm{mol}$ range in binding affinity among the four aa-tRNAs tested. Interestingly, the cocrystal structures indicate that Glu390 can make a basespecific contact with a guanine in the minor groove of the 51-63 base pair (Nissen et al. 1995, 1999). The goal of this work is to establish the thermodynamic details of this contact to determine whether the 51-63 base pair contributes to the specificity of EF-Tu for all tRNA sequences.

\section{RESULTS}

To extend the previous observations that Glu390 contributes to the specificity of the tRNA body, the binding affinities of 11 different $E$. coli aa-tRNAs were compared using both wild-type $T$. thermophilus EF-Tu and the corresponding Glu390Ala mutant. (Fig. 1; Table 1). For six of the 11 aa-tRNAs tested, the Glu390Ala mutation resulted in a $1.0 \mathrm{kcal} / \mathrm{mol}$ or greater decrease in the binding 


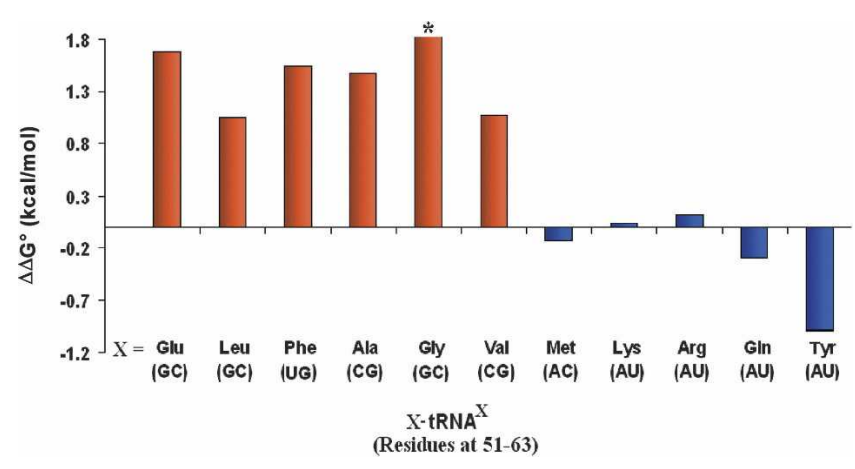

FIGURE 1. The effect of the EF-Tu Glu390Ala mutation on the binding of cognate E. coli aa-tRNAs with (red) and without (blue) a guanosine in the $51-63$ base pair. $\Delta \Delta \mathrm{G}^{\circ}$ is the $\Delta \mathrm{G}^{\circ}$ of wild-type EF-Tu minus the $\Delta \mathrm{G}^{\circ}$ for the Glu390Ala mutant protein measured in $0.5 \mathrm{M}$ $\mathrm{NH}_{4} \mathrm{Cl}, 20 \mathrm{mM} \mathrm{MgCl}_{2}, 50 \mathrm{mM}$ HEPES (pH 7.0) at $4^{\circ} \mathrm{C}$. Listed below the tRNA is the 51-63 base pair. The asterisk indicates where binding to the Glu390Ala protein was too weak to be accurately measured.

free energy. In contrast, four aa-tRNAs bound the Glu390Ala mutant with a free energy similar to native EF-Tu. Finally, in the case of Tyr-tRNA ${ }^{\text {Tyr }}$, the Glu390Ala mutation actually stabilized the binding by $1.0 \mathrm{kcal} / \mathrm{mol}$ compared to the wild-type protein. Among the 11 aatRNAs tested, mutation caused a total observed range of binding free energy of $>2.8 \mathrm{kcal} / \mathrm{mol}$. The magnitude of this effect is a substantial fraction of the $3.6 \mathrm{kcal} / \mathrm{mol}$ range of affinity observed among 19 different $E$. coli tRNAs acylated with valine (Asahara and Uhlenbeck 2002).

The sequences of the $11 E$. coli aa-tRNAs that were tested reveal a strong correlation between the presence of a guanine in the 51-63 base pair and the effect of the Glu390Ala mutation on the binding affinity. The six tRNAs with either a G51-C63, C51-G63, or U51-G63 base pair all exhibited a decrease in binding affinity, while the five tRNAs with an A51-U63 or A51-C63 base pair showed either no effect or an improvement in binding affinity. Thus, Glu390 is capable of making a thermodynamically productive contact with a guanine on either side of the 5163 base pair irrespective of whether it pairs with a cytosine or uracil. While this correlation is striking, it is clear that the results depend to some degree on elements outside the 51-63 base pair, since the magnitude of the effects are not uniform. For example, the $\Delta \Delta \mathrm{G}^{\circ}$ for tRNAs containing a G51-C63 pair range from 1.1 to $>1.8 \mathrm{kcal} / \mathrm{mol}$, and those containing an A51-U63 base pair ranged from 0 to $-1.0 \mathrm{kcal} / \mathrm{mol}$.

The available cocrystal structures of aa-tRNA with EF-Tu are consistent with the binding data. In the structure of T. aquaticus EF-Tu complexed with yeast Phe-tRNA ${ }^{\text {Phe }}$ (Fig. 2A,B), the carboxylate of Glu390 extends into the minor groove of the T-stem to within $3.4 \AA$ of the N2 amino group of G51, which could potentially form a hydrogen bond (Nissen et al. 1995). Indeed, in the unpublished structure of E. coli EF-Tu complexed with kirromycin and yeast Phe-tRNA $^{\text {Phe }}$ (PDB: 1OB2), Glu390 is within $2.7 \AA$ of G51 (Fig. 2C). In addition, as shown in Figure 2D, the structure of T. aquaticus EF-Tu bound to E. coli CystRNA ${ }^{\text {Cys }}$ shows that Glu390 is within $2.9 \AA$ of the N2 amino group of G63 (Nissen et al. 1999). Thus, this is a classic example of the sequence-specific recognition in the minor groove of a nucleic acid duplex by a protein by a mechanism that was initially predicted by Seeman et al. (1976). The amino group of guanine in a G-C base pair in a helix is nearly identical to its position in a $\mathrm{C}-\mathrm{G}$ base pair, while the $\mathrm{A}-\mathrm{U}$ and $\mathrm{U}-\mathrm{A}$ pairs lack a functional group at this location.

To further assess the contribution of the 51-63 base pair to the specificity of tRNA binding to EF-Tu, Leu-tRNA ${ }^{\text {Leu }}$ was chosen as an example of a tRNA that responds to the Glu390 mutation, but had not been cocrystallized with EF$\mathrm{Tu}$. The binding free energies of unmodified E. coli LeutRNA $^{\text {Leu }}$ and four mutations of the 51-63 base pair were determined with native EF-Tu and with the Glu390Ala mutant (Fig. 3). The tRNA mutations correspond to the four other most common 51-63 pairs present in bacterial tRNAs. Compared to the G51-C63 base pair present in tRNA $^{\text {Leu }}$, all four mutant tRNAs showed weaker binding to EF-Tu. Interestingly, a simple reversal of the base pair to C51-G63 results in a $\Delta \Delta \mathrm{G}^{\circ}$ of $0.6 \mathrm{kcal} / \mathrm{mol}$. Furthermore, as predicted by Nissen et al. (2003), the replacement of a wild-type pair with a G1-U63 wobble pair also reduces the free energy of binding $\left(\Delta \Delta \mathrm{G}^{\circ}=1.1 \mathrm{kcal} / \mathrm{mol}\right)$. It appears that these differences are solely due to Glu390 since both base-pair mutations showed a similar $\Delta \mathrm{G}^{\circ}=-8.4 \mathrm{kcal} / \mathrm{mol}$ when bound to Glu390Ala protein. Presumably all three mutations retain the contact with Glu390 and the slightly weaker affinities result from subtle changes in the position of their amino groups. As would be expected, the tRNA ${ }^{\text {Leu }}$ mutants with an A51-U63 or U51-A63 base pair showed even weaker binding due to the absence of the amino group needed to interact with Glu390. Interestingly, the Glu390Ala mutation actually stabilized binding of the A51-U63 and U51-A63 by about $0.6 \mathrm{kcal} / \mathrm{mol}$ compared to the wild-type protein. This suggests that part of the weaker binding of the wild-type protein to the A51-U63

TABLE 1. Binding of T. thermophilus EF-Tu to G51I E. coli Leu-tRNA ${ }^{\text {Leu }}$

\begin{tabular}{llc}
\hline Leu-tRNA $^{\text {Leu }}$ & EF-Tu & $\Delta \mathrm{G}^{\circ}(\mathrm{kcal} / \mathrm{mol})$ \\
\hline Wild type & Wild type & -9.7 \\
Wild type & Glu390Ala & -8.5 \\
151-C63 & Wild type & -8.6 \\
I51-C63 & Glu390Ala & -9.1 \\
\hline
\end{tabular}

Reported values were determined at $0.5 \mathrm{M} \mathrm{NH}_{4} \mathrm{Cl}$, $50 \mathrm{mM} \mathrm{HEPES}$, $\mathrm{pH} 7.0$, and $20 \mathrm{mM} \mathrm{MgCl}_{2}$ at $4^{\circ} \mathrm{C}$. Wild-type tRNA ${ }^{\text {Leu }}$ and I51-C63 variant were prepared by ligation of chemically synthesized half molecules. 
A

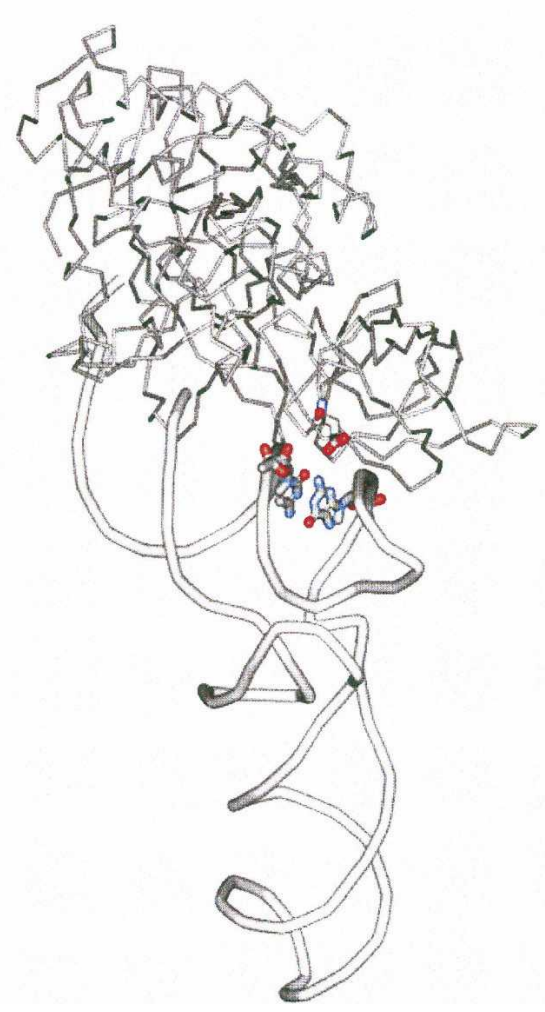

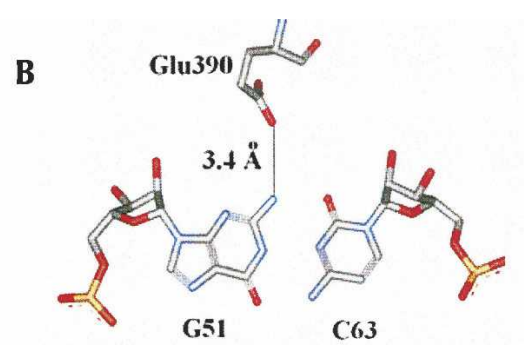

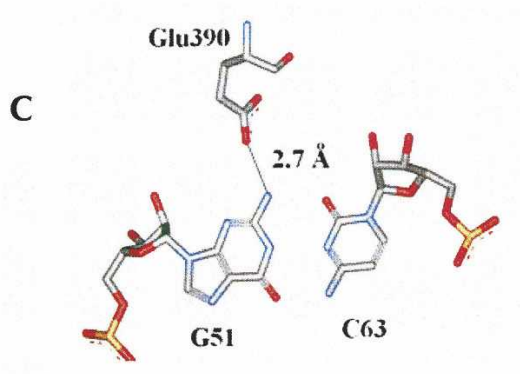

D

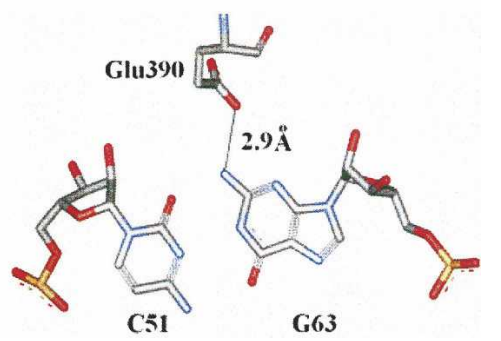

FIGURE 2. Crystallographic details of Glu390 contacting the 51-63 base pair. (A) General location and $(B)$ detail of the contact of T. aquaticus EF-Tu•GDPPNP bound to yeast Phe-tRNA ${ }^{\text {Phe }}$ (PDB:1TTT). (C) E. coli EF-Tu•GDPPNP complexed with kirromycin and yeast Phe-tRNA $^{\text {Phe }}$ (PDB: 1OB2). (D) T. aquaticus EF-Tu•GDPNP bound to E. coli Cys-tRNA ${ }^{\text {Cys }}$ (PDB: 1B23). The distances are between the caboxylate oxygen of Glu390 nearest to the amino nitrogen.

and U51-A63 tRNAs is due to a destabilizing contribution by Glu390. When the amino group is absent, the negatively charged glutamate may participate in electrostatic repulsion with the nearby phosphates and thereby act as a negative determinant.

As an alternative way to evaluate the contribution of the hydrogen bond on the stability of the complex, a variant of tRNA ${ }^{\text {Leu }}$, where G51 was replaced by an inosine, was prepared by ligating two chemically synthesized tRNA half molecules (Sherlin et al. 2001). By replacing the amino group of G51 by a proton, the I51 derivative is predicted to bind less well. As shown in Figure 3, the affinity of the I51C63 tRNA ${ }^{\mathrm{Leu}}$ is $1.1 \mathrm{kcal} / \mathrm{mol}$ weaker than the wild-type tRNA ${ }^{\text {Leu }}$, which is similar in magnitude to the U51-A63 and Glu390Ala mutations that also lack the hydrogen bond. As was observed for the A51-U63 and U51-A63 mutations, the I51-C63 tRNA binds slightly better to EF-Tu carrying the Glu390Ala mutation, again indicating the destabilizing effect of Glu390 when the amino group is absent.

Based on the preceding experiments, it appears likely that the identity of the 51-63 base pair will affect the affinity of all E. coli tRNAs to EF$\mathrm{Tu}$. Thus, it should be possible to strengthen the binding of those tRNAs that have unfavorable (A-U or $\mathrm{U}-\mathrm{A}$ ) pairs by mutating them so that they contain the G51-C63 alternative. To test this, transcripts of both wild-type and G51C63 versions of $E$. coli tRNA ${ }^{\mathrm{Arg}}{ }_{(\mathrm{UCU})}$, tRNA $^{\text {Tyr }}{ }_{(\mathrm{GUA})}$, and tRNA ${ }_{\text {(GAC) }}$ were prepared, and their binding affinities to EF-Tu were measured (Table 2). As expected, the replacement of the A51U63 base pair in tRNA ${ }^{\text {Tyr }}$ and tRNA ${ }^{\text {Arg }}$ and the U51-A63 base pair in tRNA ${ }^{\text {Val }}$ by a G51-C63 base pair stabilized binding to EF-Tu. The $\Delta \Delta \mathrm{G}^{\circ}$ values were similar to those seen for the same base pair changes in tRNA ${ }^{\text {Leu }}$ (Fig. 3), supporting the idea that a G51-C63 base pair stabilizes the binding of EF-Tu to any tRNA by a similar amount.

\section{DISCUSSION}

Using a combination of protein and tRNA mutagenesis, we have established that the interaction between $T$. thermophilus EF-Tu and the tRNA body is significantly stabilized by a hydrogen bond observed in the X-ray structure between Glu390 and the amino group of guanine in the minor groove at the 51-63 base pair in the T-stem. Hydrogen bonds between the side chains of glutamate or aspartate with the amino group of guanine are found in numerous protein-nucleic complexes (Allers and Shamoo 2001; Jones et al. 2001; Treger and Westhof 2001; Cheng et al. 2003). For example, the carboxylate of Asp235 of $E$. coli glutamanyl tRNA synthetase contacts the amino

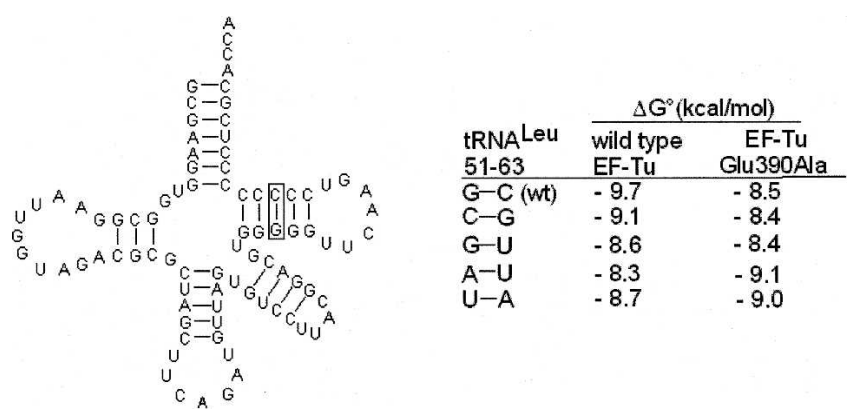

FIGURE 3. Cloverleaf representation of unmodified E. coli tRNA ${ }^{\text {Leu }}$ showing the effect of mutations of the 51-63 base pair on the binding to wild-type T. thermophilus EF-Tu and the Glu390Ala mutation. Binding experiments were performed in the same buffer as Figure 1. 
TABLE 2. Improving the $\Delta \mathrm{G}^{\circ}$ of $E$. coli aa-tRNAs by introducing G51-C63

\begin{tabular}{lccc}
\hline aa-tRNA & $\begin{array}{c}\text { Wild-type } \Delta \mathrm{G}^{\circ} \\
(51-63)\end{array}$ & $\begin{array}{c}\text { Mutant } \Delta \mathrm{G}^{\circ} \\
(51-63)\end{array}$ & $\Delta \Delta \mathrm{G}^{\circ}$ \\
\hline Tyr-tRNA $^{\text {Tyr }}$ & $-9.6(\mathrm{~A}-\mathrm{U})$ & $-11.0(\mathrm{G}-\mathrm{C})$ & -1.4 \\
Val-tRNA $^{\text {Val }}$ & $-9.7(\mathrm{U}-\mathrm{A})$ & $-11.0(\mathrm{G}-\mathrm{C})$ & -1.3 \\
Arg-tRNA $^{\text {Arg }}$ & $-9.5(\mathrm{~A}-\mathrm{U})$ & $-10.7(\mathrm{G}-\mathrm{C})$ & -1.2 \\
\hline
\end{tabular}

Reported values were determined at $0.5 \mathrm{M} \mathrm{NH}_{4} \mathrm{Cl}, 50 \mathrm{mM}$ HEPES 7.0, and $20 \mathrm{mM} \mathrm{MgCl} 2$ at $4^{\circ} \mathrm{C}$.

group of the C2-G70 base pair of tRNA ${ }^{\text {Glu }}$ (Sherlin et al. 2000) and Glu188 of yeast asparaginyl tRNA synthetase contacts the N2 amino group of the single-stranded G34 residue of E. coli tRNA ${ }^{\text {Asp }}$ (Cavarelli et al. 1993). Considering that both glutamate and aspartate are both commonly observed interacting with the N2 amino group of guanine, it is interesting that while Glu390 in EF-Tu is universally conserved in bacteria, the corresponding amino acid in the orthologous proteins in archae and eukaryotes is always an aspartate. Thus, it appears likely that a similar sequencespecific contact occurs with tRNA in all three kingdoms.

The most critical feature of the Glu390 contact is that the amount it contributes to the overall free energy of binding depends upon the identity of the 51-63 base pair. A range of $1.4 \mathrm{kcal} / \mathrm{mol}$ was observed among the binding free energies of different $51-63$ base pairs in Leu-tRNA ${ }^{\text {Leu }}$. When the tightest $\mathrm{G}-\mathrm{C}$ pair was introduced into three E. coli tRNAs containing weaker A-U or U-A pairs their binding free energy was strengthened by $1.2-1.4 \mathrm{kcal} / \mathrm{mol}$. Such a variable thermodynamic response makes this contact an important specificity element for "tuning" the affinity of different tRNA bodies for the protein. Since the free energy of binding of 20 different valylated E. coli tRNAs ranged from -8.1 to $-11.7 \mathrm{kcal} / \mathrm{mol}$ (Asahara and Uhlenbeck 2002), the Glu390 contact could potentially be responsible for nearly one half of this "tuning."

It is instructive to compare the sequences of individual E. coli tRNAs at positions 51 and 63 with the measured affinities of their bodies. Of 46 E. coli tRNAs, 26 have a guanine at this base pair, which is expected to give tighter binding. For the most part, these are indeed the tRNA bodies that bind tightly. For example, the three tightest E. coli tRNA bodies; Glu, Asp, and Gly, all contain a G51-C63, the tightest binding base pair. Meanwhile, the two weakest binding E. coli elongator tRNAs, Tyr and Gln, both have the weakest binding A51-U63 base pair. Additionally, tRNA $^{\text {Sec }}$ contains an A51-U63 pair, which may help explain why it does not bind EF-Tu in vivo (Baron et al. 1990; Ott et al. 1990a). However, there are clear exceptions, where weak binding tRNAs such as tRNA $^{\text {Trp }}$ and tRNA ${ }^{\mathrm{fMet}}$ have the very stable G51-C63 base pair. This suggests that these tRNAs must contain other sequence determinants that weaken EF-Tu binding affinity and thereby offset the stabilizing contribution of the 51-63 pair. One such determinant could be the 1-72 pair, which makes extensive contacts with EF-Tu. Indeed in the case of tRNA $^{\mathrm{fMet}}$, which does not bind EF-Tu in vivo, the unusual mismatched C1-A72 base pair has been shown to be an important "antideterminant" that weakens EF-Tu binding (Fischer et al. 1985; Seong and RajBhandary 1987). When this base pair is changed to C1-G72 or U1-A72, tRNA ${ }^{\text {fMet }}$ binds EF-Tu much better, undoubtedly aided by the stabilizing $51-63$ base pair. In the case of tRNA ${ }^{\text {Trp }}$, which must bind EF-Tu only weakly in order to offset its tight binding amino acid, it may be its A1-U72 pair that serves as the weakening element, an hypothesis that can be tested by mutagenesis experiments. It is clear that the discovery of the first tRNA specificity element for EF-Tu presented here will greatly aid in the identification of additional tRNA specificity elements.

Since Glu390 in EF-Tu is universally conserved among bacteria, it is interesting to consider whether the contact is used by all bacterial EF-Tu to modulate their affinities to their corresponding tRNAs. One way to approach this is to examine the sequence of the 51-63 base pair among bacterial tRNAs specific for a given amino acid. If it is assumed that the hierarchy of tRNA binding affinities for all bacterial EF-Tu resembles that determined for T. thermophilus EF-Tu with E. coli tRNAs (Asahara and Uhlenbeck 2002), then the tighter binding tRNAs should contain a preponderance of $G$ residues in the 51-63 base pair. As shown in Figure 4 for the tRNAs from 129

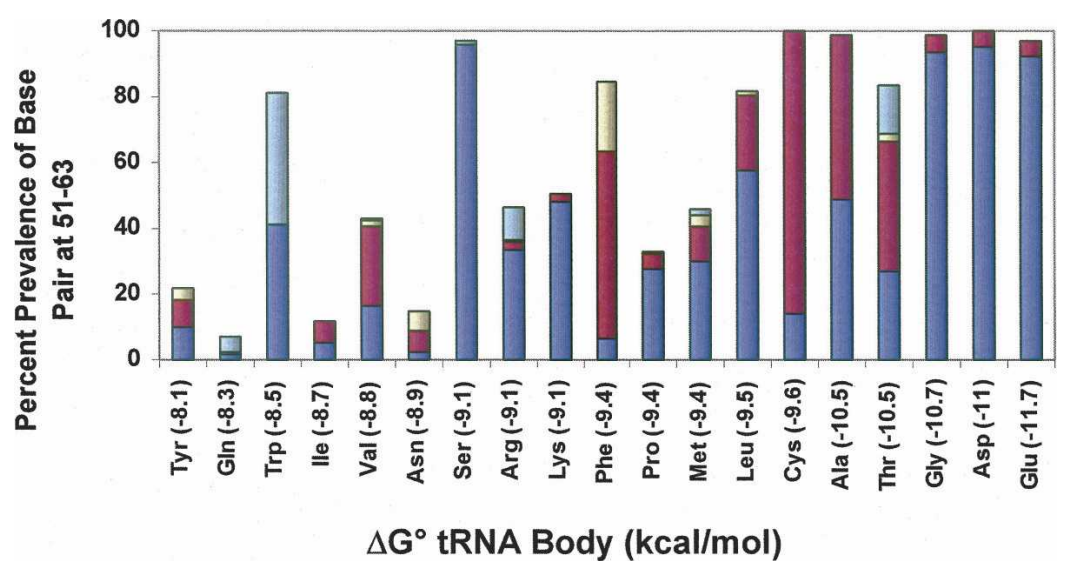

FIGURE 4. The correlation of the prevalence of guanosine in the 51-63 base pair in 129 bacterial tRNA isoacceptors with the position of the tRNA on the EF-Tu binding hierarchy. Listed in parentheses on the horizontal axis is the EF-Tu binding affinity of the tRNA body as determined for E. coli tRNAs (Asahara and Uhlenbeck 2002). Green (G51-U63), yellow (U51G63), red (C51-G63), and blue (G51-C63). 
complete bacterial genomes, this trend is clearly observed. Of the seven tightest tRNA bodies (Glu, Asp, Gly, Thr, Ala, Cys, and Leu), $>84 \%$ contain a $G$ in the $51-63$ pair while $<18 \%$ of three of the four weakest tRNA bodies (Tyr, Gln, and Ile) have a $G$ at this position. While this correlation supports the view that Glu390 "tunes" the affinity of EF-Tu for tRNA in all bacteria, the correlation is not perfect. For example, there are a limited number of bacteria where tRNA $^{\text {Glu }}$ does not contain a $G$ at 51 or 63 or where tRNA ${ }^{\text {Tyr }}$ does contain either a G51-C63 or C51-G63 pair. This could either mean that in these bacteria either Glu390 is not a specificity determinant, or the hierarchy of tRNA binding affinities is dramatically different. However, it seems more likely that the tRNAs in these organisms have evolved their sequences to use one or more of the other specificity determinants to set their appropriate binding affinities. This would partially explain why tRNA sequences are much more phylogenetically variable among bacteria than the EF-Tu sequences to which they bind.

\section{MATERIALS AND METHODS}

\section{EF-Tu mutagenesis and purification}

The initial plasmid containing the T. thermophilus tufl gene was provided by Anidya Banerjee and Marvin Mackinen (University of Chicago). A sequence coding for (His) ${ }_{6}$ TEV cleavable linker was appended to the $5^{\prime}$ end of the EF-Tu gene by PCR and cloned into a pET-3a vector between NdeI and BamHI sites. The N-terminal amino acid sequence of the resulting protein was $\mathrm{MH}_{6} \mathrm{GNKYFQA}$ so that cleavage by TEV after the glutamine resulted in the native $\mathrm{N}$-terminal alanine residue. Mutagenesis and purification of EF-Tu was performed as described previously (Sanderson and Uhlenbeck 2007a).

\section{tRNA preparation}

DNA templates for wild-type and mutant variants of E. coli $\mathrm{tRNA}^{\mathrm{Leu}}$ (CAG), $\mathrm{tRNA}^{\mathrm{Val}}$, $\mathrm{tRNA}^{\mathrm{Tyr}}$, and tRNA ${ }^{\text {Arg }}$ were prepared by primer extension using overlapping DNA oligonucleotides (IDT). In vitro transcription was performed in reactions containing $40 \mathrm{mM}$ Tris- $\mathrm{HCl}$ ( $\mathrm{pH} 8.0$ ), $10 \mathrm{mM}$ dithiothreitol, $20 \mathrm{mM}$ $\mathrm{Mg}(\mathrm{OAc})_{2}, 25 \mathrm{mM} \mathrm{NaCl}, 2 \mathrm{mM}$ spermidine, $2.5 \mathrm{mM}$ each NTP, $20 \mathrm{mM}$ GMP, $50 \mu \mathrm{g} / \mathrm{mL}$ bovine serum albumin (BSA), and $30 \mu \mathrm{g} / \mathrm{mL}$ T7 RNA polymerase. Transcribed tRNAs were purified on a $10 \%$ denaturing polyacrylamide gel. Wild-type tRNA $^{\text {Leu }}{ }_{(\mathrm{CAG})}$ and a variant containing the G51I mutation were prepared by joining tRNA half molecules using T4 tRNA ligase (Bruce and Uhlenbeck 1982; Sherlin et al. 2001). A $100 \mu \mathrm{L}$ reaction containing $45 \mu \mathrm{M}$ each of a 34 nucleotide (nt) $5^{\prime}$ half and a $53 \mathrm{nt} 3^{\prime}$ half, were combined in $10 \mathrm{mM}$ HEPES ( $\left.\mathrm{pH} 7.5\right)$ and $10 \mathrm{mM} \mathrm{KCl}$, heated to $90^{\circ} \mathrm{C}$, and allowed to cool to $40^{\circ} \mathrm{C}$ over a period of $15 \mathrm{~min}$. Subsequently, the annealed half molecules were ligated at position 34 to form a full-length tRNA by the addition of $10 \mathrm{mM}$ DTT, $20 \mu \mathrm{g} / \mathrm{mL}$ BSA, $10 \mathrm{mM}$ ATP, and $0.02 \mu \mathrm{M}$ of T4 RNA ligase to give a final volume of $112.5 \mu \mathrm{L}$. Ligation reactions were incubated at $37^{\circ} \mathrm{C}$ for $2 \mathrm{~h}$. After ligation, the full-length tRNA product was purified in a $10 \%$ denaturing polyacrylamide gel. All tRNAs were aminoacylated as described by Dale et al. (2004).

\section{EF-Tu assays}

The dissocation rate constants of aa-tRNAs from the EF-Tu•GTP complexes were determined as described previously (Sanderson and Uhlenbeck 2007a).

\section{Bioinformatic analysis}

Aligned tRNA sequences from 129 bacterial genomes were downloaded from the publicly available Web site, tRNA DataMart (http://trnamart.uoregon.edu/). The downloaded tRNA sequences represented all of the major bacterial phyla that have been identified to date. Duplicate, truncated, and/or misaligned sequences were removed. The acquired tRNA sequences were then separated according to their amino acid acceptor identity. The composition of the 51-63 base pair was then analyzed using Microsoft Excel.

\section{ACKNOWLEDGMENTS}

This grant was supported by NIH grant GM37552 to O.C.U. and also by Grant Number 1C06RR018850-01 (National Center for Research Resources [NCCR], a component of the National Institutes of Health $[\mathrm{NIH}]$ ). Its contents are solely the responsibility of the authors and do not necessarily represent the official views of NCCR or NIH.

Received January 29, 2007; accepted March 7, 2007.

\section{REFERENCES}

Allers, J. and Shamoo, Y. 2001. Structure-based analysis of proteinRNA interactions using the program ENTANGLE. J. Mol. Biol. 311: 75-86.

Asahara, H. and Uhlenbeck, O.C. 2002. The tRNA specificity of Thermus thermophilus EF-Tu. Proc. Natl. Acad. Sci. 99: 34993504.

Baron, C., Heider, J., and Bock, A. 1990. Mutagenesis of selC, the gene for the selenocysteine-inserting tRNA-species in E. coli: Effects on in vivo function. Nucleic Acids Res. 18: 6761-6766.

Bruce, A.G. and Uhlenbeck, O.C. 1982. Enzymatic replacement of the anticodon of yeast phenylalanine transfer ribonucleic acid. Biochemistry 21: 855-861.

Cavarelli, J., Rees, B., Ruff, M., Thierry, J.C., and Moras, D. 1993. Yeast tRNA ${ }^{\text {Asp }}$ recognition by its cognate class II aminoacyl-tRNA synthetase. Nature 362: 181-184.

Cheng, A.C., Chen, W.W., Fuhrmann, C.N., and Frankel, A.D. 2003. Recognition of nucleic acid bases and base pairs by hydrogen bonding to amino acid side chains. J. Mol. Biol. 327: 781-796.

Dale, T., Sanderson, L.E., and Uhlenbeck, O.C. 2004. The affinity of elongation factor Tu for an aminoacyl-tRNA is modulated by the esterified amino acid. Biochemistry 43: 6159-6166.

Fischer, W., Doi, T., Ikehara, M., Ohtsuka, E., and Sprinzl, M. 1985. Interaction of methionine-specific tRNAs from Escherichia coli with immobilized elongation factor Tu. FEBS Lett. 192: 151154.

Jones, S., Daley, D.T., Luscombe, N.M., Berman, H.M., and Thornton, J.M. 2001. Protein-RNA interactions: A structural analysis. Nucleic Acids Res. 29: 943-954.

LaRiviere, F.J., Wolfson, A.D., and Uhlenbeck, O.C. 2001. Uniform binding of aminoacyl-tRNAs to elongation factor Tu by thermodynamic compensation. Science 294: 165-168. 
Louie, A., Ribeiro, N.S., Reid, B.R., and Jurnak, F. 1984. Relative affinities of all Escherichia coli aminoacyl-tRNAs for elongation factor Tu-GTP. J. Biol. Chem. 259: 5010-5016.

Nissen, P., Kjeldgaard, M., Thirup, S., Polekhina, G., Reshetnikova, L., Clark, B.F., and Nyborg, J. 1995. Crystal structure of the ternary complex of Phe-tRNAPhe, EF-Tu, and a GTP analog. Science 270: 1464-1472.

Nissen, P., Thirup, S., Kjeldgaard, M., and Nyborg, J. 1999. The crystal structure of Cys-tRNACys-EF-Tu-GDPNP reveals general and specific features in the ternary complex and in tRNA. Structure 7: 143-156.

Nissen, P., Nyborg, J., and Clark, B.F.C. 2003. Translational elongation. In Translation mechanisms (eds. J. Lapointe and L. BrakierGingras), pp. 322-339. Kluwer Academic/Plenum Publishers, New York.

Ott, G., Jonak, J., Abrahams, I.P., and Sprinzl, M. 1990a. The influence of different modifications of elongation factor Tu from Escherichia coli on ternary complex formation investigated by fluorescence spectroscopy. Nucleic Acids Res. 18: 437-441.

Ott, G., Schiesswohl, M., Kiesewetter, S., Forster, C., Arnold, L., Erdmann, V.A., and Sprinzl, M. 1990b. Ternary complexes of Escherichia coli aminoacyl-tRNAs with the elongation factor $\mathrm{Tu}$ and GTP: Thermodynamic and structural studies. Biochim. Biophys. Acta 1050: 222-225.
Sanderson, L.E. and Uhlenbeck, O.C. 2007a. Directed mutagenesis identifies amino acid residues involved in elongation factor Tu binding to yeast Phe-tRNA ${ }^{\text {Phe }}$. J. Mol. Biol. 386: 119-130.

Sanderson, L.E. and Uhlenbeck, O.C. 2007b. Exploring the specificity of elongation factor Tu for different tRNA bodies. Biochemistry (in press).

Seeman, N.C., Rosenberg, J.M., and Rich, A. 1976. Sequence-specific recognition of double helical nucleic acids by proteins. Proc. Natl. Acad. Sci. 73: 804-808.

Seong, B.L. and RajBhandary, U.L. 1987. Mutants of Escherichia coli formylmethionine tRNA: A single base change enables initiator tRNA to act as an elongator in vitro. Proc. Natl. Acad. Sci. 84: 8859-8863.

Sherlin, L.D., Bullock, T.L., Newberry, K.J., Lipman, R.S., Hou, Y.M., Beijer, B., Sproat, B.S., and Perona, J.J. 2000. Influence of transfer RNA tertiary structure on aminoacylation efficiency by glutaminyl and cysteinyl-tRNA synthetases. J. Mol. Biol. 299: 431-446.

Sherlin, L.D., Bullock, T.L., Nissan, T.A., Perona, J.J., Lariviere, F.J., Uhlenbeck, O.C., and Scaringe, S.A. 2001. Chemical and enzymatic synthesis of tRNAs for high-throughput crystallization. RNA 7: 1671-1678.

Treger, M. and Westhof, E. 2001. Statistical analysis of atomic contacts at RNA-protein interfaces. J. Mol. Recognit. 14: 199-214. 

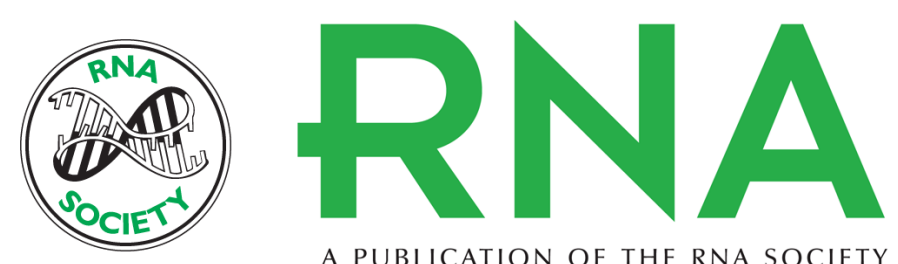

A PUBLICATION OF THE RNA SOCIETY

\section{The 51-63 base pair of tRNA confers specificity for binding by EF-Tu}

Lee E. Sanderson and Olke C. Uhlenbeck

RNA 2007 13: 835-840 originally published online April 20, 2007

Access the most recent version at doi:10.1261/rna.485307

References This article cites 21 articles, 7 of which can be accessed free at:

http://rnajournal.cshlp.org/content/13/6/835.full.html\#ref-list-1

\section{License}

Email Alerting Service

Receive free email alerts when new articles cite this article - sign up in the box at the top right corner of the article or click here.

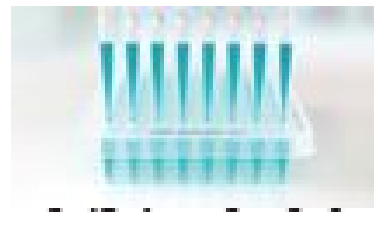

Providing Precise Solutions for your research.

To subscribe to RNA go to:

http://rnajournal.cshlp.org/subscriptions 